\title{
Glacier surges controlled by the close interplay between subglacial friction and drainage
}

\author{
Kjetil Thøgersen ${ }^{a},{ }^{*}$ Adrien Gilbert ${ }^{b}$, Coline Bouchayer ${ }^{a}$, and Thomas Vikhamar Schuler ${ }^{a}$ \\ The Njord Centre, Departments of Physics and Geosciences, University of Oslo, 0316 Oslo, Norway ${ }^{a}$ \\ Université Grenoble Alpes, CNRS, IRD, Grenoble INP, IGE, 38000 Grenoble, France
}

(Dated: March 26, 2021)

The fast flow of glaciers and ice sheets is largely influenced by friction at the icebedrock interface, and our imperfect understanding of subglacial friction accounts for one of the largest uncertainties in predictions of future sea-level rise [1]. Glacier motion ranges from slow creep to cyclic surge instabilities [2] and devastating glacier collapse [3,4] as well as continuously fast-flowing ice-streams. Glaciers dynamics also exhibits seasonal velocity variations, up-glacier and down-glacier propagation of surges, interrupted surges, as well as short-duration speed up events that do not develop into surges $[2,5,6]$. Several aspects of this wide range of glacier dynamical behavior remain elusive. This knowledge gap highlights the crucial need of developing improved descriptions of the physical processes that occur at the glacier bed as well as their couplings. Here, we show that this wide range of sliding behavior can be understood from the transient evolution of subglacial cavities and till porosity which results from a feedback loop between subglacial drainage efficiency and friction. We find potential for surging behavior at glaciers that exhibit low hydraulic conductivity at the base, together with a weak increase in hydraulic conductivity with sliding, and where the frictional response contains a transition to velocity weakening friction. This potential materializes if the local topography and surface mass balance create sufficiently thick glaciers to shut down the conduit drainage system, and where the water input to the glacier base is sufficiently high. Accurately accounting for feedback loops between friction and drainage has the potential to improve surge understanding and future assessments of hazard potential of glaciers.

\footnotetext{
* kjetil.thogersen@fys.uio.no
} 


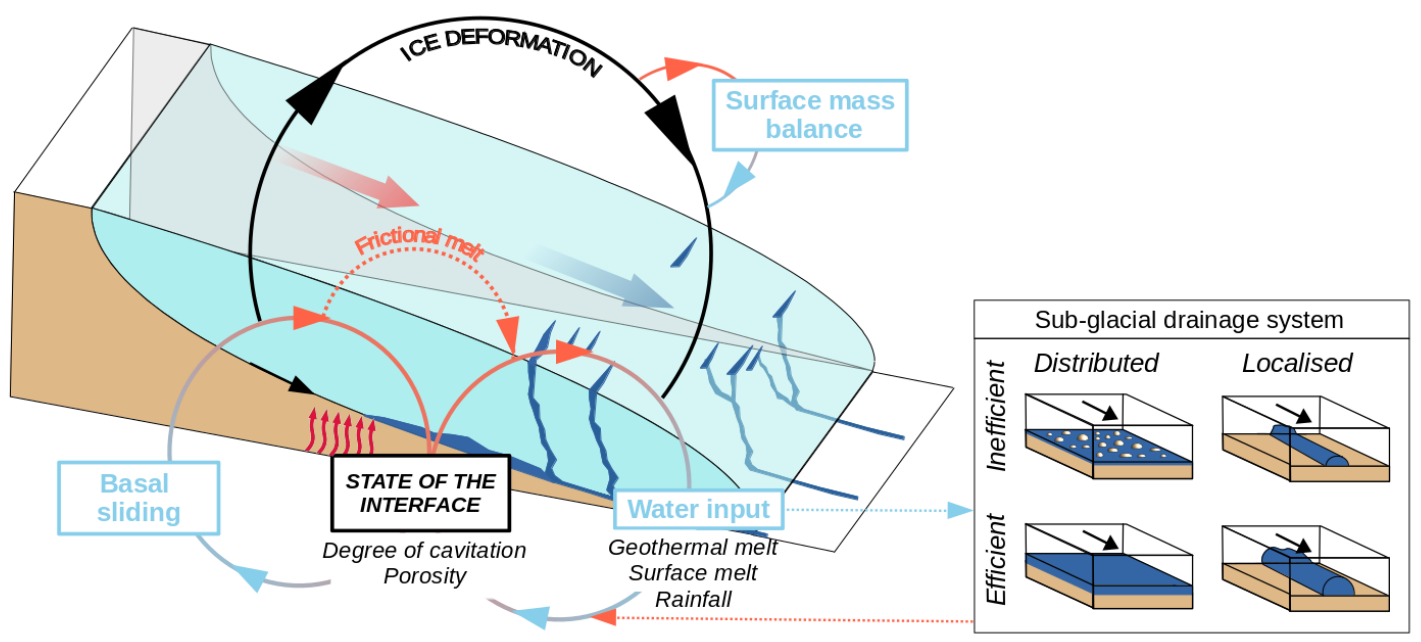

FIG. 1. Sketch of the couplings between subglacial drainage and subglacial friction. Subglacial friction and distributed drainage evolve simultaneously through a common state parameter representing the degree of cavitation or till porosity. In addition to distributed drainage which has a strong coupling with friction, the drainage system also contains localized low pressure conduits. A number of feedback loops between friction and drainage ensure the emergence of cyclic surge instabilities. The methods section contains a detailed mathematical description of these couplings, with a visual description of the friction-drainage coupling in FIG. S1 and FIG. S2.

Glacier surges are characterized by cyclic velocity variations over orders of magnitude, with fast flow typically restricted to a few years followed by a longer period of quiescence. These events feature propagation of surge fronts down-glacier, up-glacier or in both directions with an accompanying change in glacier thickness and advance of the glacier terminus $[2,6,7]$. The intervals between surges are often fairly regular $[8,9]$, but highly irregular intervals have also been reported $[10,11]$. Surging glaciers show strong geographical clustering and are most frequently found in regions in between the cold-dry and warm-humid end members [12] and are typically assumed to occur in glaciers based on deformable subglacial till, often referred to as soft-bedded glaciers [10,13, 14]. Several studies also highlighted the diversity of transient glacier dynamics that are typically not characterized as glacier surges. These range from multi-year pulsing with lower velocity increases than ordinary surges, to events that share characteristics with surge nucleation but are interrupted before they impact a large portion of the glacier $[2,15,16]$. In front of this wide spectrum of velocity variations even obtaining a robust definition of glacier surges can be difficult [16].

Historically, theories of glacier surge instabilities have been based on switches in the subglacial environment which in turn can alter glacier velocity, and several conceptual surge theories exist [17-19]. Temperate glaciers can surge due to changes in the drainage system from efficient conduits to less efficient distributed drainage, increasing water pressure and causing a surge $[17,19,20]$. Polythermal glaciers can surge due to changes in the thermal regime at the glacier base from cold to thermal allowing elevated sliding velocities [14, 18, 19]. A recent non-spatial theoretical approach overarching several earlier theories focuses on the balance between enthalpy sources and sinks [19]. This lumped model produces cyclic glacier surges, and thus provides useful insights. However, by design, non-spatial models do not predict spatial characteristics of glacier surges. The scarcity of full-scale models of glacier surges highlights the need of new approaches to understand glacier velocity variations. Here, we present such an attempt, emanating from a physically-based description of the coupling between subglacial drainage and friction. Incorporated in a glacier flow model, our approach allows to simulate surge nucleation, propagation, rapid termination, glacier advance, geometrical changes, and phenomena such as short-duration speed up events, pulsing events and interrupted surges, as well as cyclic surges, all of which spontaneously emerge from our model. The entire framework is readily available for large-scale modelling of glaciers and ice sheets.

A usual assumption is that friction couples to drainage through the effective normal stress [20-23]. However, there are strong indications that considering subglacial friction and drainage as two separate systems, that only interact through the effective normal stress, is at least in some cases not sufficient. Hard-bedded glaciers develop subglacial cavities with increasing sliding velocity, which is expected to significantly increase drainage efficiency [24]. Soft-bedded glaciers experience dilation of sediments with increasing basal motion that can alter the drainage efficiency through changes in porosity [25]. Recent theoretical and numerical developments suggest a strong influence of till dilation at the onset of glacier surges [26], and that velocity-weakening friction is a key ingredient in capturing the propagation of glacier surges [27].

To investigate the origin of the large variety of glacier flow variations, we build on the rate-and-state formulation 

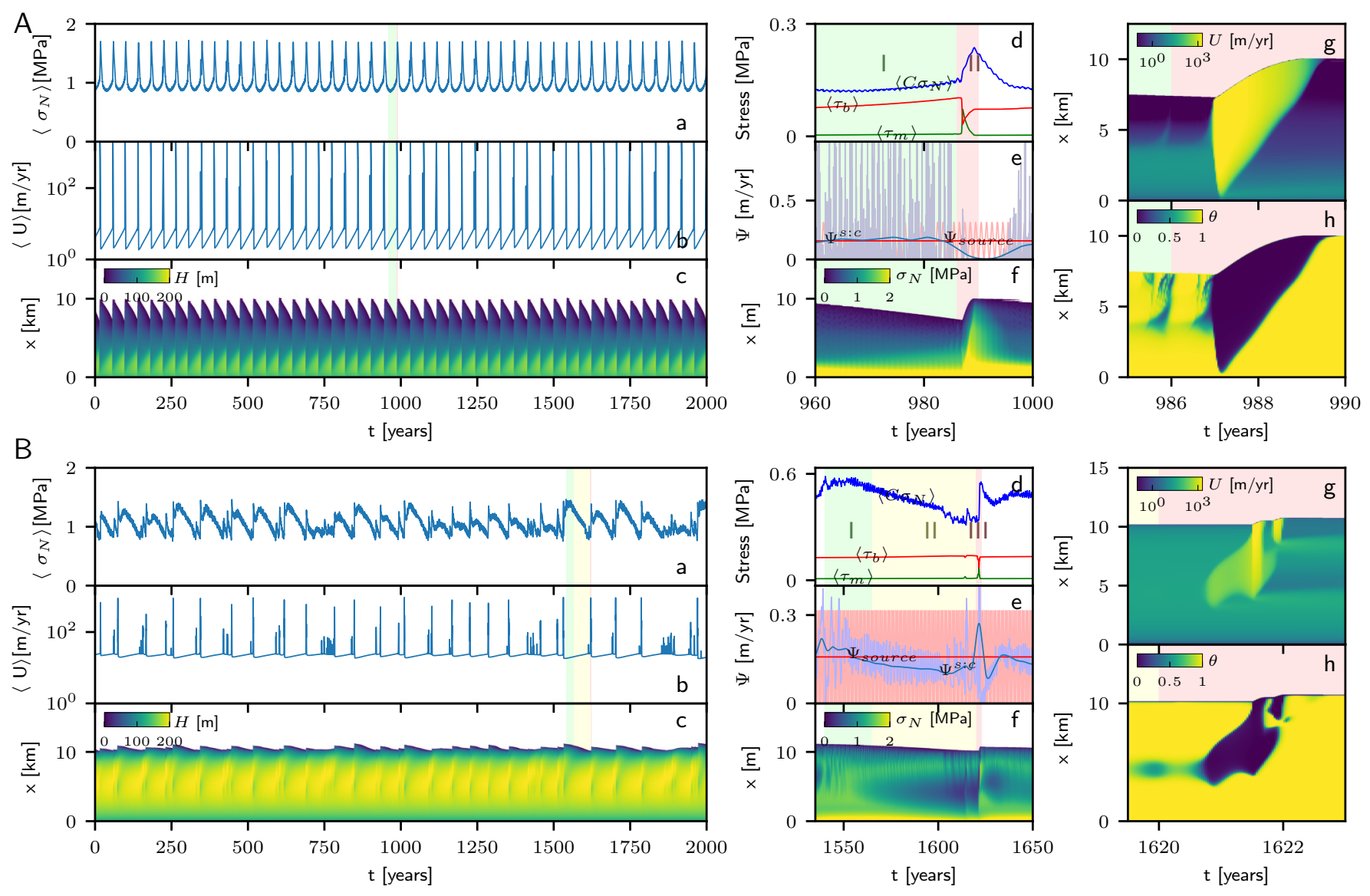

FIG. 2. A: Surges cycle governed by increasing the basal shear stress $\tau_{m}$ through mass balance-induced steepening of the glacier surface (parameter set 2 in TABLE S1). (a) Average effective normal stress $\left\langle\sigma_{N}\right\rangle$. (b) Average velocity $U$. (c) Spatiotemoral glacier thickness $H$. (d) Average effective normal stress $\left\langle\sigma_{N}\right\rangle$, basal shear stress $\left\langle\tau_{b}\right.$ and shear stress from the glacier margins $\tau_{m}$ in the quiescent phase (I) and during a surge (II). (e) Transport of water from the distributed system to the conduit system $\Psi^{s: c}$. The values are taken in the approximate trigger region $x \in[2.5,7.5] \mathrm{km}$. The faded lines show the raw data, while the deep lines show a moving gaussian average with standard deviation 2.6 years. (f) Spatiotemporal effective normal stress $\sigma_{N}$. (g) Spatiotemporal Velocity $U$ during a surge. (g) Spatiotemporal state parameter $\theta$ during a surge. B: Surges cycle governed by increasing the basal water pressure due to gradual shut-down of the conduit system (parameter set 1 in TABLE S1). (a) Average effective normal stress $\left\langle\sigma_{N}\right\rangle$. (b) Average velocity $U$. (c) Spatiotemoral glacier thickness $H$. (d) Average effective normal stress $\left\langle\sigma_{N}\right\rangle$, basal shear stress $\left\langle\tau_{b}\right.$ and shear stress from the glacier margins $\tau_{m}$ in the quiescent phase (I), during buildup of water pressure (II) and during a surge (III). (e) Transport of water from the distributed system to the conduit system $\Psi^{s: c}$. The values are taken in the approximate trigger region $x \in[2.5,7.5] \mathrm{km}$. The faded lines show the raw data, while the deep lines show a moving gaussian average with standard deviation 2.6 years. (f) Spatiotemporal effective normal stress $\sigma_{N}$. (g) Spatiotemporal Velocity $U$ during a surge. (g) Spatiotemporal state parameter $\theta$ during a surge.

used in [27], which was further developed by Gilbert et. al [28] and introduce a fully coupled rate-and-state frictiondrainage description for temperate glaciers, where both non-monotonic friction and distributed subglacial drainage are interlinked through a shared state parameter. The model dependencies are summarized in FIG. 1 and described in detail in the methods section. This coupling introduces a number of feedback loops between friction and drainage and differs from the classical approach of using friction laws that are unique and strictly increasing functions of sliding velocity and effective normal stress.

We picture drainage of water along the glacier bed in two communicating systems, a distributed system of interconnected cavities and another of arterial, low-pressure conduits. In our model, only the first one is coupled to the friction, whereas the conduit system is a highly localized feature that does not directly affect friction. Both sub-units of the drainage system have efficient and non-efficient modes, but exhibit different mechanisms for the transition between effective and ineffective drainage. The low-pressure conduits open by melting and close by viscous creep. They can efficiently drain the glacier bed as long as the normal stress is low, which means that conduit drainage efficiency reduces with increasing glacier thickness [29]. The hydraulic conductivity of the distributed drainage sys- 


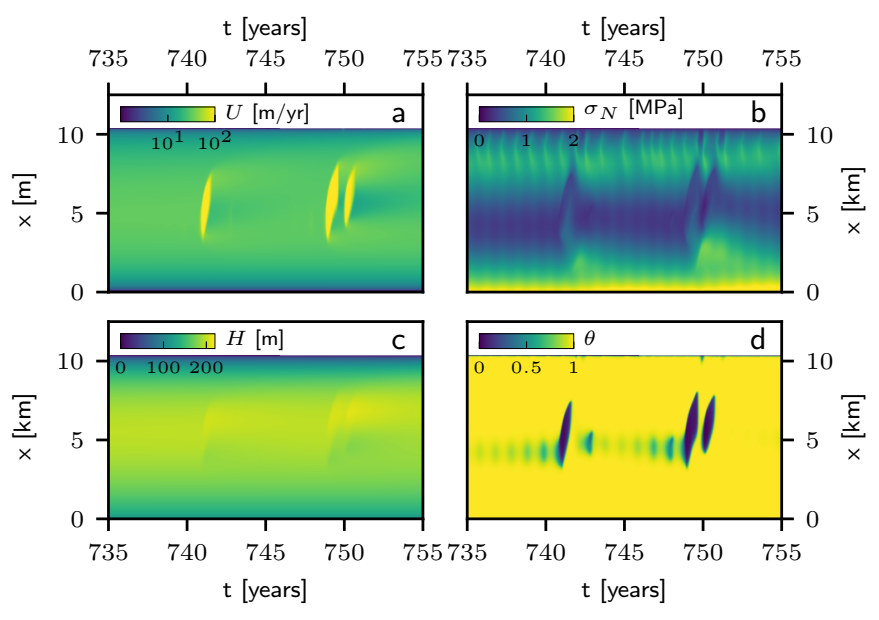

FIG. 3. The model predicts short-duration speed up events that do not develop into full surges. The hydraulic conductivity of the distributed drainage system increases when the sliding velocity increases. The temporal evolution of the drainage system then alters the distribution of effective normal stress, terminating the glacier acceleration. The figure shows spatiotemporal plots of the glacier velocity $U$ (a), the effective normal stress $\sigma_{N}$ (b), the glacier thickness $H$ (c), and the state parameter $\theta$ (d). The data shown is taken from parameter set 2 corresponding to FIG. 2 B.

tem increases with increasing sliding speed through the transient opening of interconnected subglacial cavities. This results in a strong feedback between friction and drainage which can decrease effective normal stress. In the absence of an efficient conduit system, and if the water input is sufficiently high, the only way for the glacier to evacuate excess water is to increase the hydraulic gradient, which in turn leads to an increase in both hydraulic conductivity and an increased sliding velocity. Since the change in state of the interface affects both drainage and friction simultaneously, the way the glacier modifies its sliding speed can differ drastically depending on the precise interplay between water transport at the base, the frictional response, the glacier shape, the distribution of water pressure, topography, and the interplay with subglacial conduits. The range of dynamical behaviors that we have observed in our simulations includes stable slow velocities (FIG. S5B), short duration speed-up events that do not turn into glacier-wide surges (FIG. 3), pulse-like speed-up events reminiscent of self-healing slip pulses in frictional rupture [30] (FIG. S5A) cyclic surge instabilities with close to constant recurrence intervals (FIG. 2A), as well as irregular surges where both the magnitude and the recurrence interval of the surges vary (FIG. 2B).

This large variety of glacier velocity variations somewhat challenges the definition of glacier surges. In the following, we define glacier surges as velocity increases that show a well-defined nucleation region as well as crack-like propagation (i.e. increasing extent of the surging region) accompanied by a surface bulge. In our model, these surges are triggered by a frictional instability at the glacier bed once the ratio of basal shear stress $\tau_{b}$ to effective normal stress $\sigma_{N}$ exceeds a critical value $C$ in a sufficiently large region equivalent to a nucleation length $L_{c}$ in rate-and-state friction $[27,31-$ 33]; $\tau_{b} / \sigma_{N}>C$. The instability criterion can be reached either through a combination of increased $\tau_{b}$ through an increase of the glacier surface slope and thickness with time, through an increase in water pressure at the glacier bed, or a combination thereof. In FIG. 2 we present examples of the two end-member scenarios; 1) Surges where the main mechanism is changes in the basal shear stress $\tau_{b}$ due to changes in glacier geometry (FIG. 2A), and 2) surges where the main mechanism is an increase in water pressure at the bed due to a gradual lowering of conduit drainage efficiency with increasing glacier thickness (FIG. 2B, FIG. S3).

Surges that fall into category (1) are triggered by an increase in basal shear stress with time until the glacier surface slope is sufficiently steep in a sufficiently large area of the glacier. This can in principle occur even at low water pressures with an active conduit system, given that $C$ is sufficiently low and the friction law contains a transition from velocity strengthening to velocity weakening friction. This end-member is shown in FIG. 2A, where we have selected a large value for the channel conductivity to ensure efficient conduit drainage. In the quiescent phase, the glacier surface slope increases gradually. The basal shear stress $\left\langle\tau_{b}\right\rangle$ increases gradually due to steepening of the glacier, while the average effective stress $\left\langle\sigma_{N}\right\rangle$ also increases due to thickness change (I). The water pressure remains low, as indicated by the transport of water from the distributed system to the conduit system $\Psi^{s: c}$ closely matching the water source term at the base $\Psi_{\text {source. }}$ A surge is triggered once the frictional stability threshold is surpassed. The average velocity $\langle U\rangle$ increases by around two orders of magnitude, and the surge propagates both up- and down-glacier, expanding around $2 \mathrm{~km}$. During the surge, stress is transferred from the base to the margins shown by the average shear stress on the margins $\left\langle\tau_{m}\right\rangle$ (II) This type of surge would be relatively insensitive to the hydraulic conductivity at the base, and will rely entirely on whether or not the surface mass balance can build a sufficiently steep glacier to 
trigger a frictional instability.

Surges in category (2) require inefficient subglacial conduits, which we control by decreasing the channel constant compared to scenario (1). Subglacial conduits open by melting and close by viscous creep. Since they are low pressure systems, conduits become progressively less efficient once the glacier is sufficiently thick. This gradual shut-down can manifest as a gradual increase in water pressure over several years, and the degree of subglacial cavitation changes until the hydraulic conductivity in the distributed system increases to a value that allows the water to drain. In such a case, frictional instability can be reached even for fairly large values of $C$, as along as water pressure is able to build up high enough over several years. The condition of conduit shutdown can either be met during any part of the surge cycle, or conduits could shut down at a given time prior to the surge because of changes in glacier thickness as shown in FIG. 2B. For surges in this category, the average effective normal stress $\left\langle\sigma_{N}\right\rangle$ gradually decreases in the quiescent phase, and increases after the surges when excess water is drained. In the early quiescent phase, subglacial conduits dominate the drainage (I). Prior to the surge, there is a gradual decrease of the drainage efficiency of the subglacial conduit system (II). This can be recognized by the transport of water from the distributed drainage system to the conduit system $\Psi^{s: c}$, which is gradually lowered compared to the water source term at the base $\Psi^{s}$. This causes a slow buildup of water pressure, so that the stability criterion is eventually surpassed, triggering a surge (III). The velocity increases by orders of magnitude during the surge, and in the case shown predominantly propagates down-glacier. The velocity increases significantly just before termination, and there is also a small second event after the termination of the main part of the surge. The state parameter $\theta$ decreases when velocity increases, increasing hydraulic conductivity at the glacier base. This causes a relatively rapid termination of the surge because the excess water drains as soon as the surge reaches the glacier front. This mechanism is similar to the hydraulic switch mechanism, although we stress that we do not find a hard switch but rather a gradual conduit shutdown with increasing glacier thickness.

Inter- surge intervals exhibit regular as well as irregular length (FIG. 2, FIG. S4). Due to the interaction between friction and the two types of drainage systems, irregular intervals can occur even under constant mass balance conditions where water input is only subject to seasonal variations. We have not carried out a detailed analysis of the origin of irregular surge cycles, but given the close connection between the non-linear rate-and-state friction-drainage law used here and classical rate-and-state friction both regular and chaotic solutions are to be expected (see e.g. [34]). We argue that this feature of glacier surges should be assessed when interpreting the cause of changes in surge intervals. We also stress that the separation between regular and irregular surge cycle is not expected to follow the end-member scenarios FIG. 2A and FIG. 2B, and that differences between these two simulations should not be seen as the cause of irregular intervals.

The coupling between sheet drainage and friction results in a number of different classes of non-surging glaciers. Glaciers do not surge i) if the background hydraulic conductivity is large or the water input to the glacier base is small, so that water pressure does not build up. ii) if the drainage capacity of subglacial conduits is large enough to drain all incoming water. This criterion is met if the glacier stays sufficiently thin, and links directly to the surface mass balance conditions. iii) if the hydraulic conductivity of the distributed drainage system allows for drainage of all incoming water through increased sliding velocity, but without surpassing a frictional stability threshold. Although non-surge type in the sense of the surge definition we have adopted here, this class of glaciers can show strong velocity fluctuations, seasonal variations, and pulse-like events (FIG. S5A). Note that the conditions i, ii and iii apply in combination for a glacier the surface slope of which is small enough so that the maximum friction threshold is not surpassed at low water pressures.

These criteria highlight the role of basal conditions in glacier surges. In particular, the hydraulic conductivity at the bed and its link to friction directly controls if a glacier can be surge-type or not. Glaciers where the hydraulic conductivity is either sufficiently high at low sliding speeds, or where the hydraulic conductivity can increase drastically with relatively small increases in sliding speed without triggering frictional instability will be non-surge type (given that $C$ is sufficiently large compared to the surface slope). Surges can occur for glaciers where a frictional stability threshold is surpassed in the process of increasing the hydraulic conductivity of the distributed drainage system. This means that understanding the precise interplay between friction and drainage is key to determine if a glacier can be surge type or not, given that the climatic conditions allow for it. We identify low hydraulic conductivity, combined with a limited ability to increase the hydraulic conductivity through increasing sliding speed as key factors. If combined with a transition from velocity strengthening friction at low sliding velocities to velocity-weakening friction at higher velocities this can produce surges that propagate both up- and down-glacier with well defined surge fronts. The role of the limited increase in hydraulic conductivity with increased sliding speed may indicate why most surges occur at soft-bedded glaciers. The increase in hydraulic conductivity through till dilation is expected to be limited compared to the increase of hydraulic conductivity that can occur through opening of subglacial cavities.

Climatic variables are also key factors in determining whether a glacier is of surge-type or not. FIG. 4 shows how glacier velocity variations are affected by the surface mass balance gradient (which controls the glacier thickness) and the water supply to the bed, keeping all other parameters constant (parameter set 5 in TABLE S1). We find that: i) A low water input to the bed inhibits large velocity fluctuations. ii) The limiting water input for the onset of large 


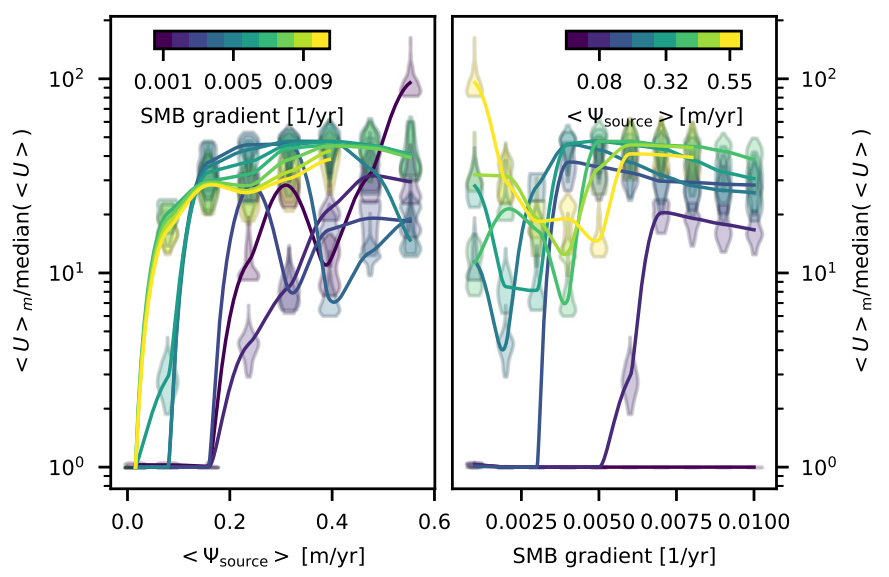

FIG. 4. Change in surge behavior with variations in climatic variables SMB gradient, and the source term $\Psi_{\text {source }}$ of water reaching the bed. All other parameters are kept constant (Parameter set 5 in TABLE S1). Each point is found from a 3000 year simulation where the violins show the probability distribution of the maxima in average velocities $\langle U\rangle_{m}$ (prominence $1 \%$ of maximum velocity and width 3.65 days) where we have excluded all maxima $\langle U\rangle_{m}<(\max (\langle U\rangle)-\min (\langle U\rangle)) / 2$. The solid lines pass the average of the selected velocity maxima.

velocity fluctuations decreases with increasing surface mass balance gradient. iii) The limiting surface mass balance gradient for the onset of large velocity fluctuations increases with decreasing water input. In turn this implies that non-surge type glaciers, given favorable basal conditions, can switch to surge-type through an increasing surface mass balance gradient, through an increased water input to the bed, or a combination thereof. Changes in surging behavior for temperate glaciers are thus expected as a result of changes in the duration and intensity of the melt-season and precipitation patterns.

Despite the ability of the model to capture a large variety of glacier velocity variations, several parameters of the friction-drainage coupling remain elusive. This coupling has so far received little experimental attention, resulting in the several model parameters used here effectively have to be treated in an empirical fashion. Experiments simultaneously measuring hydraulic conductivity and sliding speed, including transient changes, can provide very useful information in constraining the coupling between subglacial friction and drainage, for both soft-bedded and hardbedded glaciers. If combined with experimental and observational constraints on empirical parameters and functional dependencies, we believe the physical framework adopted here has the potential to significantly improve our ability to predict glacier velocity variations. Unlocking this potential opens new possibilities to improve assessments of hazard potential of glaciers, but it also offers a largely unexplored path to reduce uncertainties related to basal friction in ice sheet models that form the basis for predictions of sea-level rise.

[1] Ritz, C. et al. Potential sea-level rise from antarctic ice-sheet instability constrained by observations. Nature 528, 115-118 (2015).

[2] Cuffey, K. M. \& Paterson, W. S. B. The physics of glaciers (Academic Press, 2010).

[3] Kääb, A. et al. Massive collapse of two glaciers in western tibet in 2016 after surge-like instability. Nature Geoscience 1 (2018).

[4] Gilbert, A. et al. Mechanisms leading to the 2016 giant twin glacier collapses, aru range, tibet. The Cryosphere 12, 2883-2900 (2018).

[5] Horgan, H. J. et al. Glacier velocity variability due to rain-induced sliding and cavity formation. Earth and Planetary Science Letters 432, 273-282 (2015).

[6] Altena, B., Scambos, T., Fahnestock, M. \& Kääb, A. Extracting recent short-term glacier velocity evolution over southern alaska and the yukon from a large collection of landsat data. The Cryosphere 13, 795-814 (2019).

[7] Dunse, T. et al. Glacier-surge mechanisms promoted by a hydro-thermodynamic feedback to summer melt. The Cryosphere 9, 197-215 (2015).

[8] Meier, M. F. \& Post, A. What are glacier surges? Canadian Journal of Earth Sciences 6, 807-817 (1969).

[9] Eisen, O., Harrison, W. \& Raymond, C. The surges of variegated glacier, alaska, usa, and their connection to climate and mass balance. Journal of Glaciology 47, 351-358 (2001).

[10] Björnsson, H., Pálsson, F., Sigurðsson, O. \& Flowers, G. E. Surges of glaciers in iceland. Annals of Glaciology 36, 82-90 
(2003).

[11] Bhambri, R., Hewitt, K., Kawishwar, P. \& Pratap, B. Surge-type and surge-modified glaciers in the karakoram. Scientific reports 7, 1-14 (2017).

[12] Sevestre, H. \& Benn, D. I. Climatic and geometric controls on the global distribution of surge-type glaciers: implications for a unifying model of surging. Journal of Glaciology 61, 646-662 (2015).

[13] Hamilton, G. S. \& Dowdeswell, J. A. Controls on glacier surging in svalbard. Journal of Glaciology 42, 157-168 (1996).

[14] Jiskoot, H., Murray, T. \& Boyle, P. Controls on the distribution of surge-type glaciers in svalbard. Journal of Glaciology 46, 412-422 (2000).

[15] Turrin, J. B., Forster, R. R., Sauber, J. M., Hall, D. K. \& Bruhn, R. L. Effects of bedrock lithology and subglacial till on the motion of ruth glacier, alaska, deduced from five pulses from 1973 to 2012. Journal of Glaciology 60, 771-781 (2014).

[16] Herreid, S. \& Truffer, M. Automated detection of unstable glacier flow and a spectrum of speedup behavior in the alaska range. Journal of Geophysical Research: Earth Surface 121, 64-81 (2016).

[17] Fowler, A. A theory of glacier surges. Journal of Geophysical Research: Solid Earth 92, 9111-9120 (1987).

[18] Fowler, A., Murray, T. \& Ng, F. Thermally controlled glacier surging. Journal of Glaciology 47, 527-538 (2001).

[19] Benn, D., Fowler, A. C., Hewitt, I. \& Sevestre, H. A general theory of glacier surges. Journal of Glaciology 65, 701-716 (2019).

[20] Flowers, G. E. Modelling water flow under glaciers and ice sheets. Proceedings of the Royal Society A: Mathematical, Physical and Engineering Sciences 471, 20140907 (2015).

[21] Iken, A. The effect of the subglacial water pressure on the sliding velocity of a glacier in an idealized numerical model. Journal of Glaciology 27, 407-421 (1981).

[22] Walder, J. S. Hydraulics of subglacial cavities. Journal of Glaciology 32, 439-445 (1986).

[23] Kamb, B. Glacier surge mechanism based on linked cavity configuration of the basal water conduit system. Journal of Geophysical Research: Solid Earth 92, 9083-9100 (1987).

[24] Hoffman, M. \& Price, S. Feedbacks between coupled subglacial hydrology and glacier dynamics. Journal of Geophysical Research: Earth Surface 119, 414-436 (2014).

[25] Clarke, G. K. Subglacial till: a physical framework for its properties and processes. Journal of Geophysical Research: Solid Earth 92, 9023-9036 (1987).

[26] Minchew, B. \& Meyer, C. R. Dilation of subglacial sediment governs incipient surge motion in glaciers with deformable beds. Proceedings of the Royal Society A 476, 20200033 (2020).

[27] Thøgersen, K., Gilbert, A., Schuler, T. V. \& Malthe-Sørenssen, A. Rate-and-state friction explains glacier surge propagation. Nature communications 10, 1-8 (2019).

[28] Gilbert, A., Gimbert, F., Thøgersen, K., Schuler, T. V. \& Kääb, A. Consistent framework for coupling basal friction with subglacial hydrology on hard-bed glaciers. In preparation (2021).

[29] Meierbachtol, T., Harper, J. \& Humphrey, N. Basal drainage system response to increasing surface melt on the greenland ice sheet. Science 341, 777-779 (2013).

[30] Shlomai, H. \& Fineberg, J. The structure of slip-pulses and supershear ruptures driving slip in bimaterial friction. Nature communications 7, 1-7 (2016).

[31] Rice, J. \& Ruina, A. L. Stability of steady frictional slipping. Journal of applied mechanics 50, 343-349 (1983).

[32] Scholz, C. H. Earthquakes and friction laws. Nature 391, 37 (1998).

[33] Handwerger, A. L., Rempel, A. W., Skarbek, R. M., Roering, J. J. \& Hilley, G. E. Rate-weakening friction characterizes both slow sliding and catastrophic failure of landslides. Proceedings of the National Academy of Sciences 113, 10281-10286 (2016).

[34] Erickson, B., Birnir, B. \& Lavallée, D. A model for aperiodicity in earthquakes. Nonlinear processes in geophysics 15, 1-12 (2008).

[35] Bueler, E. \& Brown, J. Shallow shelf approximation as a ?sliding law? in a thermomechanically coupled ice sheet model. Journal of Geophysical Research: Earth Surface 114 (2009).

[36] Nick, F. M., Van der Veen, C. J., Vieli, A. \& Benn, D. I. A physically based calving model applied to marine outlet glaciers and implications for the glacier dynamics. Journal of Glaciology 56, 781-794 (2010).

[37] Gagliardini, O., Cohen, D., Råback, P. \& Zwinger, T. Finite-element modeling of subglacial cavities and related friction law. Journal of Geophysical Research: Earth Surface 112 (2007).

[38] Zoet, L. K. \& Iverson, N. R. Experimental determination of a double-valued drag relationship for glacier sliding. Journal of Glaciology 61, 1-7 (2015).

[39] Zoet, L. K. \& Iverson, N. R. Rate-weakening drag during glacier sliding. Journal of Geophysical Research: Earth Surface 121, 1206-1217 (2016).

[40] Werder, M. A., Hewitt, I. J., Schoof, C. G. \& Flowers, G. E. Modeling channelized and distributed subglacial drainage in two dimensions. Journal of Geophysical Research: Earth Surface 118, 2140-2158 (2013).

[41] Zoet, L. K. \& Iverson, N. R. A slip law for glaciers on deformable beds. Science 368, 76-78 (2020). 


\section{METHODS}

\section{Governing equations}

To facilitate simulations over thousands of years with time-steps in the range of seconds to minutes, we use a flowline model [35], which combines the integrated shallow shelf approximation (SSA) and the shallow ice approximation (SIA). The SIA velocity is found assuming no sliding at the base and constant temperature. The stress balance where the driving force due to gravity is balanced by shear deformation.

$$
u_{\text {SIA }}=-2(\rho g)^{n}|\nabla h|^{n-1} A \frac{(h-b)^{n+1}}{n+1} \nabla h .
$$

Here, $y$ is the vertical coordinate $\rho$ is the ice density, $g$ is the gravitational acceleration, $n$ is Glen's exponent, $h$ is the altitude, $A$ is the ice rheology constant, which we consider to be constant, and $b$ is the bedrock topography. Below we will use the definition

$$
D_{\mathrm{SIA}}=2(\rho g)^{n}|\nabla h|^{n-1} A \frac{(h-b)^{n+2}}{n+2}
$$

Sliding is accounted for by the SSA solution

$$
\begin{array}{r}
\nabla \cdot\left(2 A^{-1 / n} H\left|\nabla u_{\mathrm{SSA}}\right|^{\frac{1}{n}-1} \nabla u_{\mathrm{SSA}}\right) \\
-\tau_{b}\left(u_{\mathrm{SSA}}\right)-\tau_{m}\left(u_{\mathrm{SSA}}\right)-\rho g H \nabla h=0
\end{array}
$$

where $\tau_{b}$ is the frictional stress explained below, and $H$ is the thickness. In addition to the friction force at the base we include an approximation of stress from the glacier margins (e.g. [36])

$$
\tau_{m}=\frac{2 H}{W}\left(\frac{5 u_{\mathrm{SSA}}}{A W}\right)^{\frac{1}{3}}
$$

The SIA and SSA solutions are coupled through

$$
U=\left(1-f\left(\left|u_{\mathrm{SIA}}\right|\right)\right) u_{\mathrm{SIA}}+f\left(\left|u_{\mathrm{SSA}}\right|\right) u_{\mathrm{SSA}}
$$

where we adopt the same empirical function as Bueler [35]

$$
f(|u|)=1-\frac{2}{\pi} \arctan \left(\frac{u^{2}}{100[\mathrm{~m} / \mathrm{yr}]^{2}}\right)
$$

For mass continuity we solve the advection diffusion equation

$$
\begin{aligned}
\frac{\partial h}{\partial t} & =\nabla \cdot\left(\left(1-f\left(\left|u_{\mathrm{SIA}}\right|\right)\right) D_{\mathrm{SIA}} \nabla h\right) \\
& -\nabla \cdot\left(f\left(\left|u_{\mathrm{SSA}}\right|\right) u_{\mathrm{SSA}} H\right)+\mathrm{SMB}
\end{aligned}
$$

We base the friction law on the newly developed rate-and-state friction formulation for temperate beds [27], and then couple it with the subglacial drainage through the state parameter $\theta$. The state of the interface $\theta$ determines both the frictional stress and the hydraulic conductivity through transient opening of subglacial cavities. The state evolution law can be written as

$$
\frac{\partial \theta}{\partial t}=\frac{v}{d_{c}}\left(\theta^{\dagger}-\theta\right)+\frac{1}{t_{c}}\left(\theta^{\dagger}-\theta\right)
$$

where $d_{c}$ is a length scale on the order of the characteristic cavity size, and $t_{c}$ is a characteristic time-scale expected to be related to ice rheology and the typical cavity size, with a lower limit set by the Maxwell time of ice. The length scale was used in [27], but in order to account for cavity opening and closure due to water pressure variations that could occur at low sliding speeds a time-scale is needed. First, the basal shear stress is found through the state parameter

$$
\tau_{b}=\theta\left(\frac{v}{A_{s}}\right)^{1 / m}
$$


where $A_{s}$ is a friction prefactor and $m$ is a creep exponent. In steady state, at the pressure melting point, the basal shear stress is given by the parameterization by Gagliardini et al. [37]

$$
\tau_{b, \mathrm{ss}}=\sigma_{N} C\left(\frac{\chi}{1+\alpha \chi^{q}}\right)^{1 / m}
$$

where $C$ determines the maximum friction threshold in steady state (Iken's bound), $\sigma_{N}$ is the effective normal stress, $q$ is an exponent that determines the degree of velocity weakening friction, $\chi=\frac{v}{C^{m} \sigma_{N}^{m} A_{s}}$, and $\alpha=\frac{(q-1)^{q-1}}{q^{q}}$. A transition from velocity strengthening to velocity-weakening friction for hard bed glaciers has also been verified experimentally [38]. The state parameter in steady state can be found by combining equation 9 and 10 and is given by

$$
\theta^{\dagger}=\left(\frac{1}{1+\alpha \chi^{q}}\right)^{1 / m}
$$

For the normal stress we assume that the effective normal stress is positive, i.e. we do not solve for additional uplift due to water pressure exceeding the overburden pressure. In determining the frictional stress, this approximation has no practical significance as the limit of zero effective normal is $\tau_{b}=0$.

A few comments on the rationale behind the rate-and-state formulation are in order. Equation 9 for $\theta=1$ is the limit of the steady state solution when $v \rightarrow 0$. In this limit, there is no direct normal stress dependency on basal shear stress because there is no cavitation. Using equation 9 requires the assumption that the basal shear stress depends on the configuration of subglacial cavities, which in steady state depends on normal stress and sliding velocity. With the additional assumption that the low velocity limit is independent of normal stress because there is no additional cavities forming when you increase the sliding speed, the natural conclusion is that for a step change in velocity where cavities have no time to develop there will be a strain hardening following the form of the low velocity limit. This will be modified by a factor in $[0,1]$ because there is not necessarily full contact between ice and bedrock in this case. The form of equation 9 has experimental support, and follows closely the observations by Zoet et. al [39]. In addition to the strain-hardening with stepwise increases in velocity (FIG. S1) that has been directly observed experimentally [39], equation 9 introduces a transient response in basal shear stress as the effective normal stress changes (FIG. S2). This transient response is a direct consequence of the assumption that the basal shear stress depends on cavity configuration. To our knowledge this effect has not been investigated in experiments or simulations. Although this transient response arises through the same assumptions that cause the transient response with changes in velocity, this lack of direct experimental evidence means it is subject to some degree of uncertainty. The same type of uncertainty applies for the state evolution law in equation 8. Physical arguments can give us approximate values for the characteristic length and time-scales involved. While we find it unlikely that $d_{c}$ and $t_{c}$ are truly constant across the entire range of $\theta, \sigma_{N}$ and $v$, treating them as constant is likely acceptable as a first order estimate.

The drainage system is split in two components; distributed drainage through a sheet, and localized conduit drainage. Assuming laminar flow in the sheet, discharge is given by [20]

$$
Q_{s}(\phi, \theta)=-K(\theta) \nabla \phi
$$

where $\phi$ is the hydraulic potential, and the hydraulic conductivity $K$ is determined by the amount of cavity opening through the empirical relation

$$
K(\theta)=K_{0}+K_{\mathrm{s}}(1-\theta)^{3} f_{\text {perc }}(\theta)
$$

Here, $K_{0}$ is the background conductivity in the limit of zero cavity opening, $K_{\text {sheet }}$ is the maximum conductivity when the cavities are fully open. $f_{\text {perc }}(\theta)$ is a function accounting for drainage percolation that takes values between 0 and 1, i.e. the hydraulic conductivity increases rapidly then the cavities start to connect.

In order to perform simulations over thousands of years we use an approximate solution of conduits by assuming that the water pressure in the conduits $p_{w, c}=0$. This assumption allows us to avoid solving for pressure variations within the conduits, and instead solve for the water exchange between the conduits and the distributed drainage system. This allows for a larger time-step in the simulations. Discharge through conduits is given by [20]

$$
Q_{\mathrm{c}}=-k_{\mathrm{c}} S^{\alpha_{c}}\left|\nabla \phi_{c}\right|^{\beta_{c}-2} \nabla \phi
$$

where $k_{c}$ is a channel constant, $S$ is the cross-sectional area, $\phi_{c}$ is the hydraulic potential in the sheet, and $\alpha_{c}$ and $\beta_{c}$ are exponents accounting for turbulent flow[20]. The conduits open by melting, and close by viscous creep [20]. Under the assumption of $p_{w, c}=0$, the evolution of the conduit cross sectional area $S$ can be written as 


$$
\frac{\partial S}{\partial t}=\frac{\left(\left|Q_{\mathrm{c}}\right|+\left|\Delta_{\mathrm{c}} Q_{\mathrm{s}}\right|\right)\left|\nabla \phi_{c}\right|}{\rho_{\text {ice }} L}-2 S\left(\frac{\sigma_{n}}{n B}\right)^{n}
$$

where $\Delta_{c}$ is the distance between conduits, $\phi_{c}=\rho_{w} g b$ is the hydraulic potential in the conduits at zero water pressure, $n$ is Glen's exponent, $\sigma_{n}$ is the normal stress at the base, $L$ is latent heat, and $B$ is a ice rheology constant which determines how fast conduits close. A correction term is to the water pressure needed because conduits contain a solution where they grow indefinitely, draining water from the sheet at negative water pressures. To remove this solution from the equations, we increase the closure term $B$ of the conduits by a factor $10^{3}$ when the water pressure in the sheet is negative. The exchange source term between the two systems required to keep $p_{w, c}=0$ is then given by

$$
\Delta_{\mathrm{c}} \Psi^{s: c}=\frac{\partial S}{\partial t}+\nabla \cdot Q_{c}-\frac{\left(\left|Q_{\mathrm{c}}\right|+\left|\Delta_{\mathrm{c}} Q_{\mathrm{s}}\right|\right) \rho g|\nabla b|}{\rho_{\text {water }} L}
$$

Following [40] we solve for the hydraulic potential in a distributed drainage system

$$
\frac{e_{v}}{\rho_{w} g} \frac{\partial \phi}{\partial t}+\nabla \cdot Q_{s}(\phi, \theta)+h_{0} \frac{\partial \theta}{\partial t}+\Psi_{\text {source }}+\Psi_{\text {melt }}+\Delta_{c} \Psi^{c: s}=0
$$

where $e_{v}$ is an aquifer void ratio, $h_{0}$ is a thickness constant relating the state parameter to a physical sheet thickness, $\Psi_{\text {source }}$ is a combined water source term from rainfall and surface melt reaching the bed, as well as melting due to geothermal heat. $\Psi_{\text {melt }}$ is a frictional melt rate $\Psi_{\text {melt }}=\frac{U_{\mathrm{SSA}} \tau_{m}}{L \rho_{\mathrm{ice}}}$.

It should be noted that the mathematical framework presented here is based on experimental and numerical evidence on hard bedded glaciers. In the manuscript, we have allowed for parameter values of hydraulic conductivity that are also within the range of what is expected for soft-bedded glaciers. In this limit, the state parameter is likely more representative of till porosity than the degree of subglacial cavitation, such as in e.g. [26]. Soft-bedded glaciers also have a maximum friction threshold often called the Coulomb criterion. In the limit of $q=1$, the mathematical formulation is expected to be similar for hard-bedded and soft-bedded glaciers [41], but we stress that it should be seen as empirical in this range of hydraulic conductivities.

\section{Description of simulations}

We set up synthetic glaciers and integrate the combined set of equations using an implicit time-stepping algorithm with adaptive time-stepping with maximum time-step of $10^{3} \mathrm{~s}$. We first solve the system with an SIA solver assuming no sliding for 500 years, and then run it for a further 1000 years with the coupled SIA/SSA solver to reach steady conditions.

Parameter sets are given in tables S2 and S1. Most simulations use the same topography given by

$$
h(x)=2000 e^{-x / 15000 \mathrm{~m}},
$$

but the frictional triggered surge in FIG. 2A has a slight modification in topography compared to the rest of the simulations

$$
h(x)=2000 e^{-x / 15000 \mathrm{~m}}-10 \operatorname{atan}\left(\frac{x-7000 \mathrm{~m}}{500 \mathrm{~m}}\right) .
$$

The reason for this is to make sure the largest slope of the glacier bed more or less coincides with the maximum surface slope, and that this occurs in the central part of the glacier. This design allows us to set the nucleation region of the surge to the central part of the glacier.

The climatic forcing is set through the surface mass balance and the water input to the bed. The surface mass balance term is assumed to be constant (i.e. we neglect seasonal variations), and linear in altitude. The gradient of the surface mass balance term is varied systematically in FIG. 4. The water supply to the bed includes seasonal variations through a sinusoidal dependence with a period of one year. 

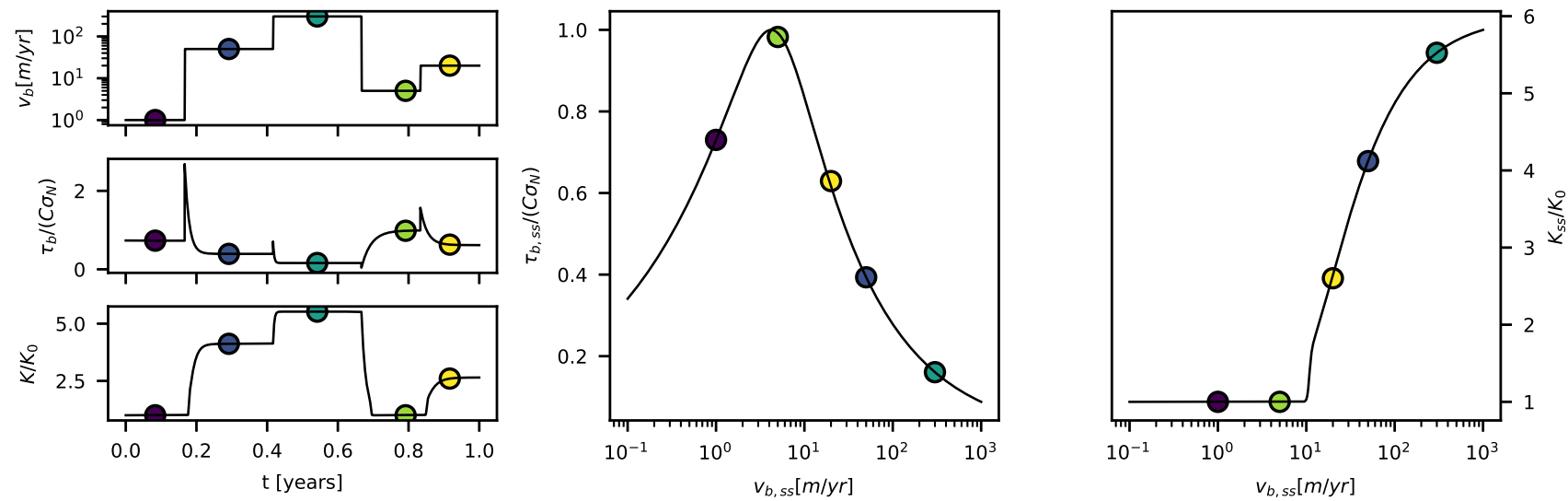

359

365

FIG. S1. Response of the rate-and-state friction law to step changes in sliding speed. Markers are consistent throughout the panels. Top left: Sliding speed as a function of time. Middle left: Basal shear stress as function of time. Bottom left: Hydraulic conductivity as a function of time. Middle: Steady state basal shear stress as a function of velocity. Right: Steady state hydraulic conductivity as a function of velocity. Parameters used: $\sigma_{N}=0.5 \mathrm{MPa}, q=2.5, m=3, C=0.4, d_{c}=1 \mathrm{~m}, t_{c}=10^{6} \mathrm{~s}$, $A_{s}=10^{-23} \mathrm{~s}^{-1} \mathrm{~Pa}^{-3}$.
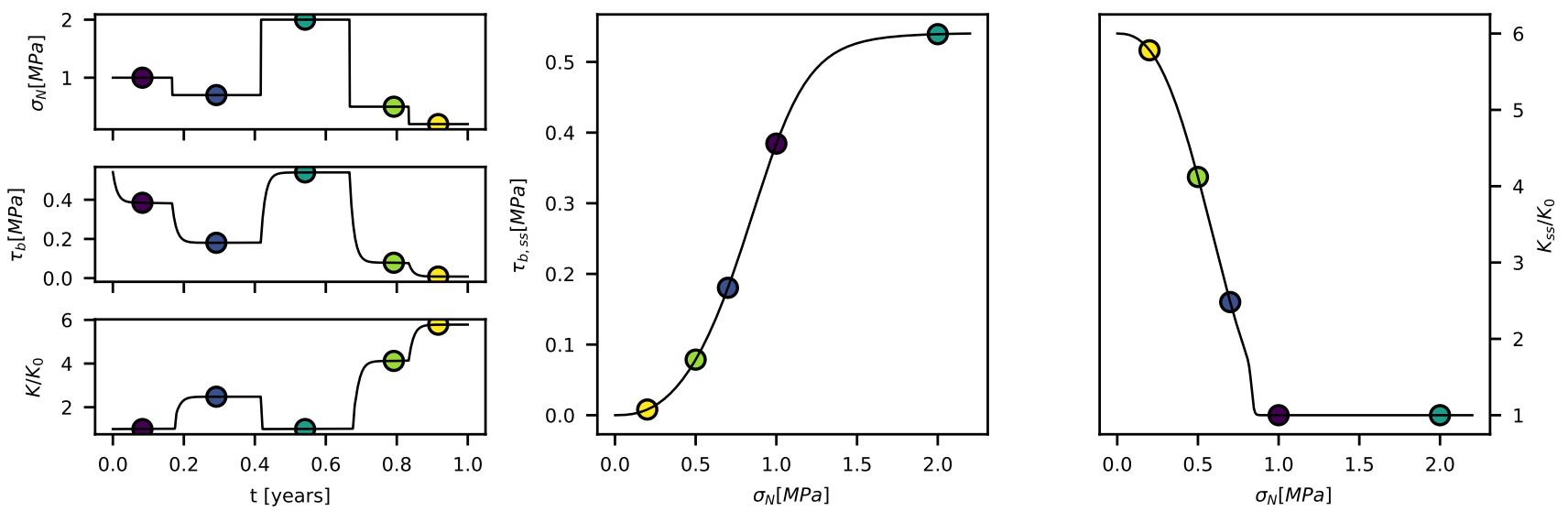

FIG. S2. Response of the rate-and-state friction law to step changes in effective normal stress. Markers are consistent throughout the panels. Top left: Effective normal stress as a function of time. Middle left: Basal shear stress as function of time. Bottom left: Hydraulic conductivity as a function of time. Middle: Steady state basal shear stress as a function of effective normal stress. Right: Steady state hydraulic conductivity as a function of effective normal stress. Parameters used: $v_{b}=50 \mathrm{~m} / \mathrm{yr}$, $q=2.5, m=3, C=0.4, d_{c}=1 \mathrm{~m}, t_{c}=10^{6} \mathrm{~s}, A_{s}=10^{-23} \mathrm{~s}^{-1} \mathrm{~Pa}^{-3}, K_{0}=10^{-8} \mathrm{~m} / \mathrm{s}, K_{s}=5 \times 10^{-8} \mathrm{~m} / \mathrm{s}$. 


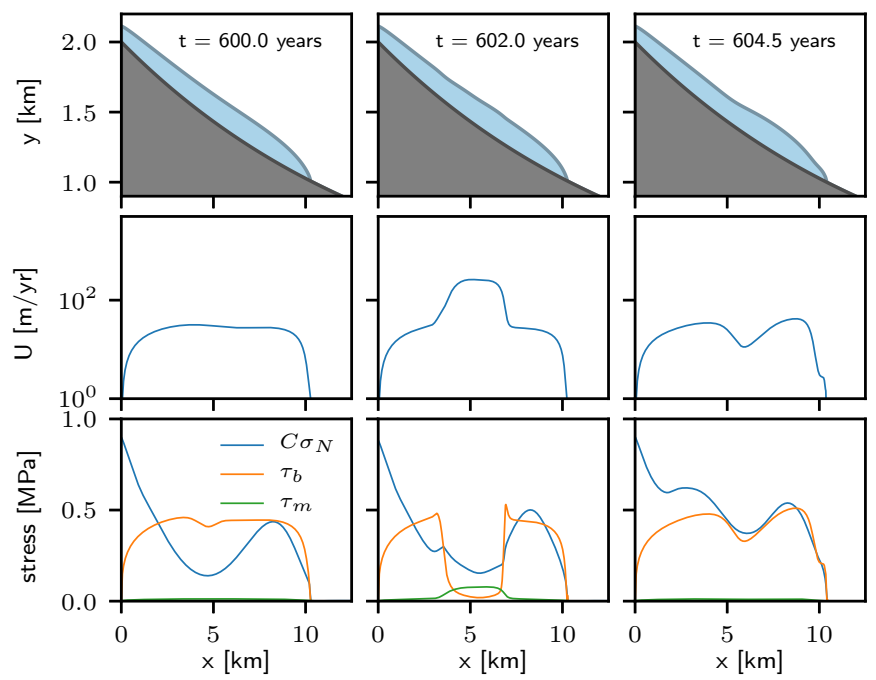

FIG. S3. Glacier geometry, velocity $U$, effective normal stress $\sigma_{N}$, basal shear stress $\tau_{b}$ and margin stress $\tau_{m}$ at nucleation (left), during (middle) and after (right) a surge in the model (corresponding to FIG. 2B). The figure is available as a movie in the supplemental materials.

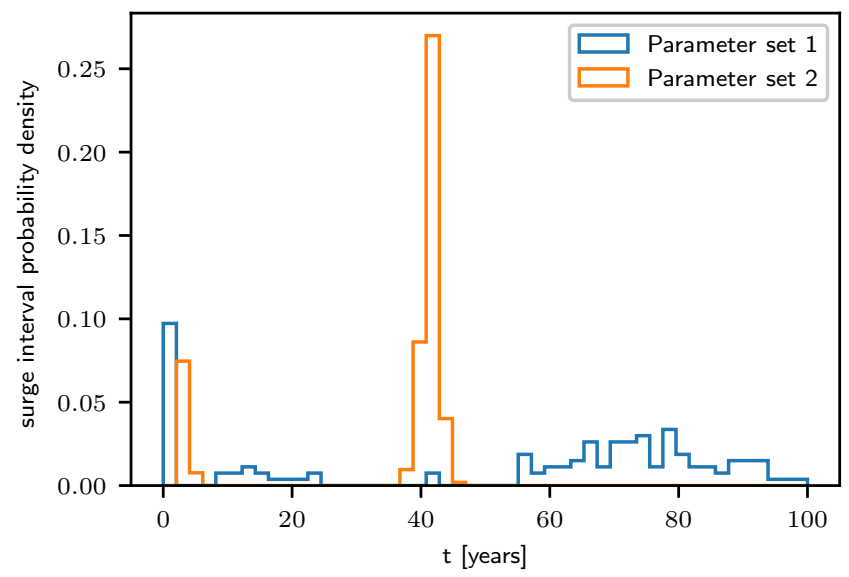

FIG. S4. Surge intervals corresponding to the surges in FIG. S3. The surges intervals are found from defining a glacier as surging when $\langle U\rangle>100 \mathrm{~m} / \mathrm{yr}$. The data is measured from 9000 years of simulation time. 

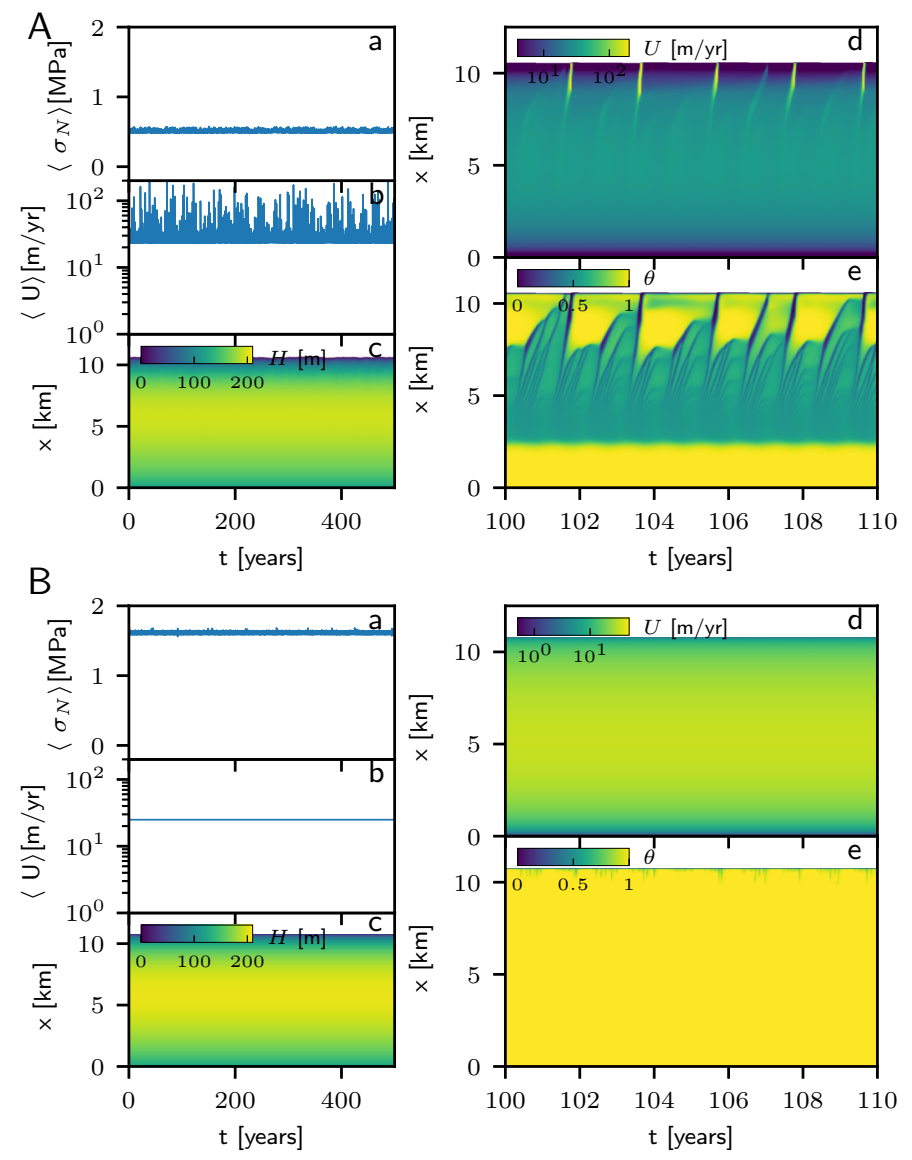

FIG. S5. Two classes of non-surging glaciers that are non-surge type because of an efficient distributed drainage system (A, parameter set 4 TABLE S1), or efficient conduit system (B, parameter set 3 TABLE S1)). A shows strong velocity variations due to the coupling between sliding velocity and distributed drainage. B shows a close to constant sliding velocity because the water pressure is kept low at all times. a: Average effective normal stress $\left\langle\sigma_{N}\right\rangle$. b: Bverage velocity $\langle U\rangle$. c: Spatiotemporal glacier thickness $H$. d: Spatiotemporal velocity $U$. e: Spatiotemporal state parameter $\theta$. (a-e applies to both A and B). 


\begin{tabular}{|c|c|c|c|c|c|c|c|}
\hline Parameter & Description & Units & 1 & 2 & 3 & 4 & 5 \\
\hline$h(x)$ & Bed topography & {$[\mathrm{m}]$} & equation 18 & equation 19 & equation 18 & equation 18 & equation 18 \\
\hline$k_{c}$ & Channel conductivity & {$\left[\mathrm{m}^{3 / 2} / \mathrm{kg}^{1 / 2}\right]$} & 0.003 & 0.1 & 0.1 & 0.001 & 0.003 \\
\hline$C$ & Maximum friction threshold & [] & 0.4 & 0.14 & 0.4 & 0.4 & 0.4 \\
\hline$\Psi_{\text {source }}$ & Water source term & {$[\mathrm{m} / \mathrm{s}]$} & $10^{-8} \sin ^{2}\left(\frac{\pi t}{\mathrm{yr}}\right)$ & $10^{-8} \sin ^{2}\left(\frac{\pi t}{\mathrm{yr}}\right)$ & $10^{-8} \sin ^{2}\left(\frac{\pi t}{\mathrm{yr}}\right)$ & $10^{-8} \sin ^{2}\left(\frac{\pi t}{\mathrm{yr}}\right)$ & $\Psi_{0} \sin ^{2}\left(\frac{\pi t}{\mathrm{yr}}\right)$ \\
\hline$S M B(y)$ & Surface mass balance & {$[\mathrm{m} / \mathrm{s}]$} & $\frac{4 \times 10^{-3}(y-1600 \mathrm{~m})}{1 y r}$ & $\frac{4 \times 10^{-3}(y-1600 \mathrm{~m})}{1 y r}$ & $\frac{4 \times 10^{-3}(y-1600 \mathrm{~m})}{1 \mathrm{yr}}$ & $\frac{4 \times 10^{-3}(y-1600 \mathrm{~m})}{1 y r}$ & $\mathrm{SMB}_{0}(y-1600 \mathrm{~m})$ \\
\hline$K_{s}$ & Hydraulic conductivity distributed & {$[\mathrm{m} / \mathrm{s}]$} & $5 \times 10^{-8}$ & $2 \times 10^{-7}$ & $1 \times 10^{-6}$ & $1 \times 10^{-6}$ & $5 \times 10^{-8}$ \\
\hline$h_{0}$ & Characteristic drainage thickness & {$[\mathrm{m}]$} & 0.1 & 0.01 & 1.0 & 1.0 & 0.1 \\
\hline$W$ & Glacier width & {$[\mathrm{m}]$} & 1500 & 1500 & 1500 & 1500 & 1200 \\
\hline
\end{tabular}

TABLE S1. List of parameters that are varying for different simulations in the manuscript, split in different sets.

\begin{tabular}{|l|c|c|c|}
\hline Parameter & Description & Units & value \\
\hline$\rho_{\text {ice }}$ & ice density & {$\left[\mathrm{kg} / \mathrm{m}^{3}\right]$} & 900 \\
$\rho_{\text {water }}$ & water density & {$\left[\mathrm{kg} / \mathrm{m}^{3}\right]$} & 1000 \\
$g$ & Gravitational acceleration & {$\left[\mathrm{m} / \mathrm{s}^{2}\right]$} & 9.8 \\
$L$ & Latent heat of fusion & {$[\mathrm{J} / \mathrm{kg}]$} & $3 \times 10^{5}$ \\
$m$ & Friction law rheology exponent & {[]} & 3 \\
$n$ & Glen's exponent & {[]} & 3 \\
$\beta_{c}$ & Conduit drainage exponent & {[]} & $3 / 2$ \\
$\alpha_{c}$ & Conduit drainage exponent & {[]} & $5 / 4$ \\
$A$ & Ice rheology constant & {$[1 /(\mathrm{sPa})]$} & $2.4 \times 10^{-24}$ \\
$\Delta_{c}$ & Conduit spacing & {$[\mathrm{m}]$} & 1000 \\
$d_{c}$ & characteristic cavity opening length scale & {$[\mathrm{m}]$} & 1.0 \\
$t_{c}$ & characteristic cavity closure time scale & {$[\mathrm{s}]$} & $5 \times 10^{6}$ \\
$B$ & Conduit closure term & {$\left[\mathrm{Pa} / \mathrm{s}^{1 / 3}\right]$} & $10^{8}$ \\
$A_{s}$ & Basal shear stress prefactor & $\left.\left[\mathrm{m} /(\mathrm{sPa})^{3}\right)\right]$ & $10^{-23}$ \\
$W$ & Glacier width & {$[\mathrm{m}]$} & 1500 \\
$f_{\text {perc }}$ & Percolation function & {[]} & $\frac{1}{2}\left(\tanh \left(50\left(\frac{1}{2}-\theta\right)\right)+1\right)$ \\
$e_{v}$ & Aquifer void ratio & {[]} & $10^{-2}$ \\
$q$ & Friction decay exponent & {[]} & 2.5 \\
$K_{0}$ & Background hydraulic conductivity & {$[\mathrm{m} / \mathrm{s}]$} & $10^{-8}$ \\
\hline
\end{tabular}

TABLE S2. List of parameters that are kep constant for all simulations presented in the manuscript. 